\title{
Transmigrated neutrophils in the intestinal lumen engage ICAM-1 to regulate the epithelial barrier and neutrophil recruitment
}

\author{
R Sumagin, AZ Robin, A Nusrat and CA Parkos
}

Neutrophil (PMN) transepithelial migration (TEM) and accumulation in luminal spaces is a hallmark of mucosal inflammation. TEM has been extensively modeled; however, the functional consequences and molecular basis of PMN interactions with luminal epithelial ligands are not clear. Here we report that cytokine-induced expression of a PMN ligand, intercellular adhesion molecule-1 (ICAM-1), exclusively on the luminal (apical) membrane of the intestinal epithelium results in accumulation and enhanced motility of transmigrated PMN on the apical epithelial surface. Using complementary in-vitro and in-vivo approaches, we demonstrate that ligation of epithelial ICAM-1 by PMN or with specific antibodies results in myosin light-chain kinase-dependent increases in epithelial permeability that are associated with enhanced PMN TEM. Effects of ICAM-1 ligation on epithelial permeability and PMN migration in vivo were blocked after intraluminal addition of peptides derived from the cytoplasmic domain of ICAM-1. These findings provide new evidence for functional interactions between PMN and epithelial cells after migration into the intestinal lumen. Although such interactions may aid in clearance of invading microorganisms by promoting PMN recruitment, engagement of ICAM-1 under pathologic conditions would increase accumulation of epithelial-associated PMN, thus contributing to mucosal injury as observed in conditions, including ulcerative colitis.

\section{INTRODUCTION}

During mucosal inflammation, neutrophil (PMN) infiltration of epithelial surfaces leads to injury and leaky mucosal barrier. Such barrier defects underlie the basis of a number of inflammatory disorders. For example, accumulation of PMN in the alveolar space and in epithelial intestinal crypts has been shown to directly correlate with the severity of diseases such as acute lung injury, ${ }^{1,2}$ cystic fibrosis ${ }^{3}$ and inflammatory bowel diseases, ulcerative colitis, and Crohn's disease. ${ }^{4,5}$ PMN migration across epithelial layers and into luminal spaces is a sequential process, beginning with the extravasation of PMN from blood vessels, ${ }^{6}$ migration through the interstitium, and terminating with transmigration across the epithelium in a basolateral-to-apical (luminal) direction. Interactions necessary for initial engagement of PMN with the basolateral surface of the intestinal epithelium are primarily mediated by the PMN $\beta_{2}$-integrin CD11b/CD18 (Mac-1) ${ }^{7,8}$ and other yet unidentified ligands. These initial interactions have been shown to trigger intracellular signaling events leading to increased epithelial permeability, thus facilitating enhanced PMN transepithelial migration (TEM). Specifically, PMN contact with basolateral intestinal epithelial cell (IEC) ligands has been shown to activate protease-activated receptors- 1 and -2 , leading to enhanced phosphorylation of myosin light-chain kinase (MLCK) and a subsequent increase in epithelial permeability. ${ }^{9}$

Following initial basolateral adhesion, PMN migrating across epithelial monolayers engage in adhesive interactions with adherens and tight junctional protein complexes, ${ }^{10-12}$ and other epithelial ligands such as $\mathrm{CD} 47,{ }^{13}$ before finally arriving at the luminal (apical) epithelial membrane. Here the PMN remains in contact with the epithelial surface, and collections of apically associated PMN in the intestinal crypts constitute a pathognomonic feature of the classic crypt abscess. ${ }^{14}$

PMN-epithelial cell interactions during the late stages of TEM have recently come into focus with the identification of several apically expressed epithelial PMN ligands. Specifically, 
expression of the PMN interacting proteins, CD55, ${ }^{15} \mathrm{CD} 44,{ }^{16}$ and CD54 intercellular adhesion molecule-1 (ICAM-1), ${ }^{17}$ have been shown to be increased under inflammatory conditions. Importantly, CD44 and CD55 (decay accelerating factor) have been reported to have roles in facilitating PMN detachment from the apical surface after the completion of TEM, ${ }^{18,19}$ whereas ICAM-1 has been shown to mediate PMN adhesion through binding to Mac- $1 .^{20}$ In addition to mediating PMNepithelial cell adhesion, ligands expressed on the apical epithelial surface have also been shown to modulate epithelial homeostasis through signaling events. In particular, CD44associated signaling events have been implicated in regulating junctional composition and cell proliferation. ${ }^{21}$

ICAM-1 on endothelial cells has been previously shown to have a key role in regulating leukocyte transendothelial migration. $^{22,23}$ Moreover, ICAM-1 on endothelial cells has been shown to associate with cytoskeletal proteins and participate in cytoskeletal and junctional reorganization. ${ }^{24,25}$ ICAM-1 is markedly upregulated in the epithelium of colonic biopsies from ulcerative colitis and Crohn's disease patients, ${ }^{26}$ as well as in IEC cell lines, including T84 and Caco2, after stimulation with pro-inflammatory cytokines. ${ }^{17,27}$ Although ICAM-1 binding to PMN Mac-1 can facilitate migration in the non-physiological apical-to-basolateral direction, ${ }^{27}$ the role for ICAM-1 in PMN TEM in the physiological basolateral-toapical direction is unknown as are the epithelial functional responses to such binding events.

In this study, we used in-vitro and in-vivo approaches to investigate the functional role of ICAM-1 during the final stages of PMN TEM. We show that ICAM-1 expression on the apical epithelial surface results in enhanced PMN adhesion after TEM. Furthermore, we show that apically associated PMN exhibit increased locomotion that is ICAM-1 and Mac-1 dependent. Importantly, we show that ligation of ICAM-1 at the apical epithelial membrane leads to MLCK-dependent actomyosin contractility resulting in compromised barrier function, thereby facilitating enhanced PMN recruitment across the intestinal epithelium.

\section{RESULTS}

ICAM-1 promotes PMN adhesion and locomotion on the apical epithelial membrane under the conditions of inflammation

In crypt abscesses, as observed in active IBD, PMN infiltrating the intestinal crypts are often observed in intimate contact with the apical (luminal) epithelial surface. ${ }^{14}$ To examine PMN-IEC interactions during the late stages of TEM, we modeled PMN migration across the intestinal epithelium under inflammatory conditions by using a previously described transwell setup. ${ }^{19}$ PMN TEM in the physiological basolateral-to-apical direction across control or interferon- $\gamma$ (IFN $\gamma$ )-treated T84 IECs was induced by the addition of a transepithelial $N$-formyl-Met-LeuPhe (fMLF) gradient $(100 \mathrm{nM})$. Exposure of T84 IECs to IFN $\gamma\left(100 \mathrm{Uml}^{-1}, 24 \mathrm{~h}\right)$ had no significant effect on IEC barrier function or expression and subcellular localization of key junctional proteins JAM-A, Occludin, and ZO-1
(Supplementary Figure S1 online). IFN $\gamma$ treatment had no significant effect on the number of PMN that completed TEM; however, it significantly increased the number of apically associated PMN (from $8.7 \pm 1.8 \%$, untreated, to $19.7 \pm 1.9 \%$, IFN $\gamma$, apical, Figure 1a). Consistent with this increase, the number of non-migrated PMN (PMN in the upper chamber, basal) was significantly reduced $(\sim 1.5$-fold $)$ after IFN $\gamma$ treatment, suggesting an increased rate of migration (Figure 1a). The number of $\mathrm{PMN}$ within the epithelial monolayer (epith) was not changed. Representative confocal immunofluorescence images (Figure $\mathbf{1 b}$, upper panels) and $z$-projections (bottom panels) show apically associated PMN after migration across IFN $\gamma$ stimulated, but not control T84 monolayers. As IFN $\gamma$ has been previously shown to induce ICAM-1 expression in inflamed epithelium, ${ }^{27}$ we hypothesized that during inflammation, PMN migrating across epithelial monolayers remained adherent to the apical membrane through Mac-1- and ICAM-1-dependent interactions, and therefore would be capable of ICAM-1-dependent locomotion as has been previously observed in vascular endothelium. ${ }^{24,28}$ Confirming previous findings, IFN $\gamma$ treatment $\left(100 \mathrm{U} \mathrm{ml}^{-1}, 24 \mathrm{~h}\right)$ induced a robust, time-dependent increase in ICAM-1 expression (peaking 24 h post treatment, Figure 1c,d) on the apical membrane of T84 IECs (Figure 1e). This effect was specific to IFN $\gamma$, as exposure of T84 and SKCO15 IECs to tumor necrosis factor- $\alpha$ (TNF $\alpha$; $10 \mathrm{ng} \mathrm{ml}^{-1}$ ) failed to induce ICAM-1 expression (Supplementary Figure S2A,B). ICAM-1 upregulation was also observed in the crypt epithelium of colonic biopsies from patients with active ulcerative colitis compared with healthy mucosa (Figure 1f). Further, confirming ICAM-1- and Mac-1dependent PMN adhesion to apical IEC membranes, addition of either anti-ICAM-1 or anti-Mac-1 function-blocking antibodies (Abs; $20 \mu \mathrm{g} \mathrm{ml}^{-1}$ ) reversed the IFN $\gamma$-induced increase in PMN adhesion (Supplementary Figure S3A). Interestingly, inhibition of PMN Mac-1 resulted in a more potent reduction ( $>3$-fold) in PMN adhesion compared with inhibition of ICAM-1, suggesting that Mac-1 binds other epithelial surface ligands in addition to ICAM-1.

We next examined whether apically adhered PMN after crossing the epithelium exhibited apical locomotion. Phasecontrast time-lapse microscopy was used to track and quantify the behavior of individual PMN attached to the IEC apical membrane. PMN in the absence of exogenous stimulus exhibited little movement; however, $59.3 \pm 7.6 \%$ of apically associated PMN after migration across IECs exhibited a highly motile behavior (Figure 2a) with average crawling distances of $65.6 \pm 6.2 \mu \mathrm{m}$ (Figure $2 \mathrm{~b}$ ). TEM induced a robust increase in Mac-1 expression on the PMN cell surface (Figure 2c), consistent with its role in PMN-IEC apical interactions. Importantly, the number of PMN exhibiting apical locomotion was significantly increased when IECs were stimulated with IFN $\gamma$ to upregulate ICAM-1 ( $88 \pm 3.6 \%$, IFN $\gamma$, vs. $59.3 \pm 7.6 \%$, untreated IEC). Under these conditions, PMN traveled for significantly longer distances $(101 \pm 10.0 \mu \mathrm{m}$, IFN $\gamma$, vs. $65.6 \pm 6.2 \mu \mathrm{m}$, untreated IECs, Figure $2 \mathbf{b}$ ) along the apical epithelial membrane. Confirming the role for ICAM-1 in 
a
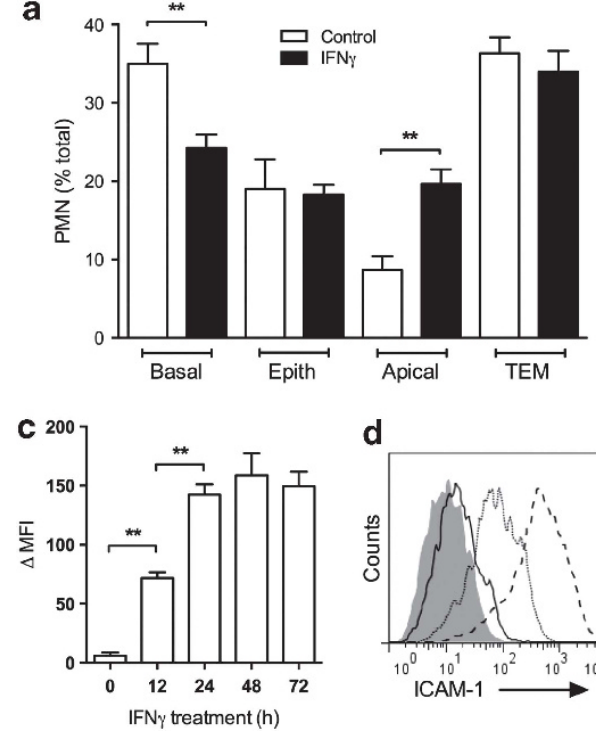

d

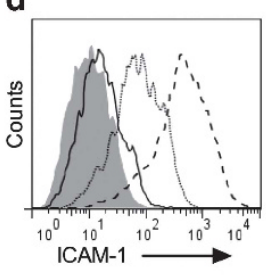

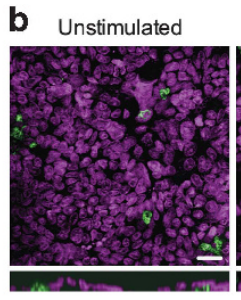

e

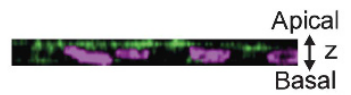

f
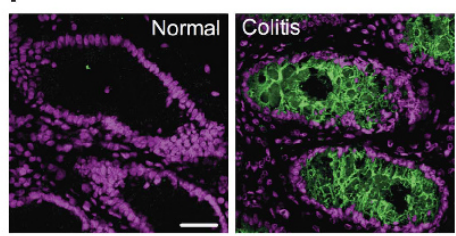

Figure 1 Interferon- $\gamma$ (IFN $\gamma$ )-induced expression of intercellular adhesion molecule-1 (ICAM-1) promotes neutrophil (PMN) retention on the apical epithelial membrane. (a) PMN was stimulated (100 nм N-formyl-Met-Leu-Phe (fMLF)) to migrate across control or IFN $\gamma$-treated T84 intestinal epithelial cells (IECs). Non-migrated PMN (basal), PMN within the epithelial layer (epith), PMN associated with the apical IEC membrane after transepithelial migration (TEM) (apical), and PMN that completed TEM (TEM) were quantified. (b) Following $1 \mathrm{~h}$ of TEM, IEC monolayers were fixed and stained for cell nuclei (blue) and PMN Mac-1 (green). Representative images show enhanced apical attachment of PMN after migration across IFN $\gamma$-treated T84 IECs. Bar $=20 \mu \mathrm{m}$. Bottom panels are projections of images acquired in series in the $z$ direction. (c-f) Confluent T84 IECs were treated with IFN $\gamma\left(100 \mathrm{U} \mathrm{ml}^{-1}, 24 \mathrm{~h}\right)$ to induce surface expression of ICAM-1. (c) Representative flow cytometry diagram and (d) quantification of ICAM-1 expression in response to IFN $\gamma$ treatment. ICAM-1 expression was induced in a time-dependent manner peaking at $24 \mathrm{~h}$. (e) T84 IECs were fixed in ethanol and immunofluorescently labeled for ICAM-1. Representative image ( $z$ projection of seven confocal slices) demonstrates that ICAM-1 expression is restricted to the apical epithelial surface. (f) Non-inflamed and inflamed tissue sections from biopsies of human patient with ulcerative colitis were stained for ICAM-1 (green) and nuclei (Topro-3, blue). Representative immunofluorescence images show the upregulation of ICAM-1 expression in inflamed tissue (right panel) but not in normal tissue (left panel). Bar $=50 \mu \mathrm{m}$. $N=5$ independent experiments in triplicates, ${ }^{* *} P<0.01$, two-tailed Student's $t$-test (a), analysis of variance with Newman-Keuls multiple comparison test (d).

mediating PMN locomotion, addition of a function-blocking anti-ICAM-1 antibody $(\mathrm{Ab})$ reversed the IFN $\gamma$ effects, reducing both the number and the distances traveled by PMN $(61.4 \pm 7.8 \%$ and $73.6 \pm 6.1 \mu \mathrm{m}$, respectively, Figure $2 \mathbf{a}, \mathbf{b})$. Inhibition of Mac-1 abolished locomotion of the remaining adherent PMN (Figure 2a and Supplementary Movies S1 and S2), confirming an exclusive role for Mac-1 in this process. The effects of Mac-1 inhibition on PMN locomotion are further highlighted in time-lapse image sequences (Figure 2d) and by displacement trajectories of six representative PMN (Figure 2e). PMN crawling velocities ranged from 3 to $7 \mu \mathrm{m} \mathrm{min}^{-1}$ and were not significantly different on unstimulated vs. IFN $\gamma$-stimulated IECs (Supplementary Figure S3B).

\section{PMN adhesion to the apical epithelial membrane compromises epithelial barrier function}

We next examined the effects of PMN interactions with apically expressed epithelial ligands on epithelial barrier function. In these experiments, PMN were added apically to confluent T84 IECs cultured on permeable supports $(0.4 \mu \mathrm{m}$ pore size, too small to allow PMN TEM), and TER, as an index of epithelial barrier, was measured over 12-h under specified conditions. The 12-h time period was selected to avoid the effects of apoptotic PMN on the epithelial barrier. ${ }^{29}$ fMLF (100 nM)- stimulated PMN adhesion to T84 IECs triggered a timedependent decrease in TER over a 12 -h time period $(\sim 60 \%$, Figure 3a). Importantly, addition of PMN to the apical membrane of T84 IECs that were pretreated with IFN $\gamma$, which we have shown leads to enhanced ICAM-1-dependent PMN adhesion (Figure 1a), resulted in a further decrease in TER ( $\sim 40 \%$ first $2 \mathrm{~h}$ and $\sim 90 \%$ over $12 \mathrm{~h}$ ). IFN $\gamma$ treatment alone had no effect on TER. To test whether the observed increase in epithelial permeability was due to a direct contact between epithelial cells and PMN, we used an anti-Mac-1 inhibitory Ab (CBRM1/29, $20 \mu \mathrm{g} \mathrm{ml}^{-1}$ ) to inhibit PMN adhesion to IFN $\gamma$ stimulated IECs. Inhibition of Mac-1 significantly attenuated the PMN-induced decrease in TER after IFN $\gamma$ stimulation (Figure 3a). Importantly, in separate experiments we further confirmed that PMN-induced decrease in TER in both unstimulated and IFN $\gamma$-treated epithelial monolayers was dependent on direct contact between PMN and the apical epithelial membrane, and not mediated through paracrine mechanisms. In such experiments, PMNs were added to the lower chamber of transwells containing inverted (apical side facing down) T84 monolayers and stimulated with fMLF $(100 \mathrm{nM})$. Under these conditions, there was no effect of PMN on the epithelial barrier as assessed by TER (Figure $\mathbf{3 b}$ ). Exposure of epithelial monolayers to $100 \mathrm{nM}$ fMLF for $12 \mathrm{~h}$ had no significant effect on TER. 

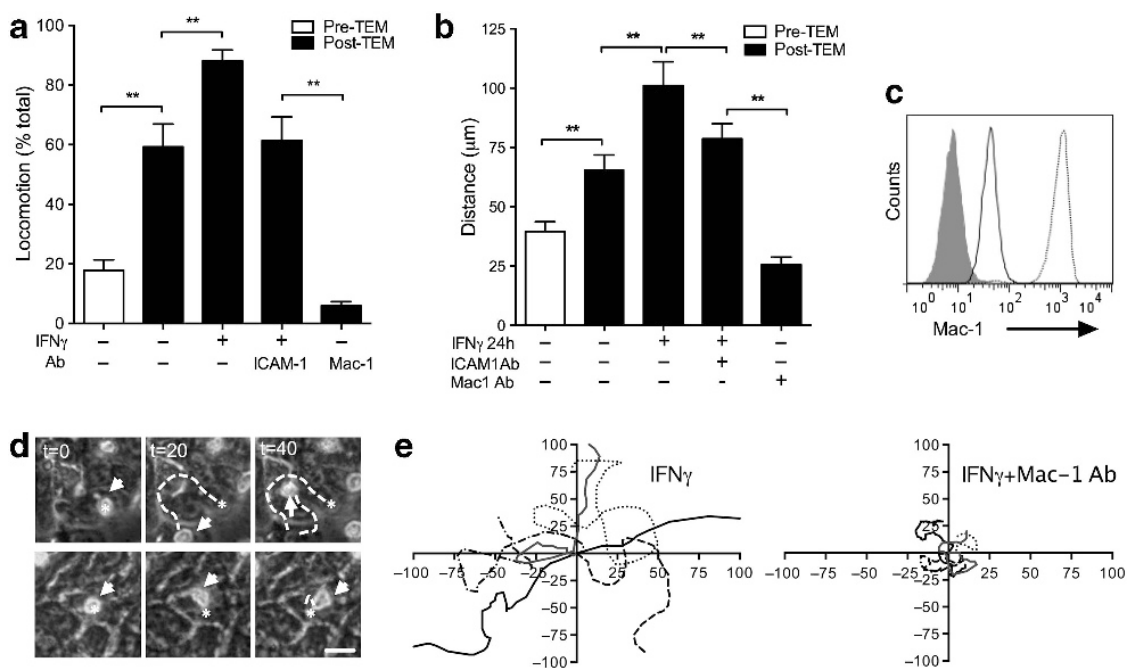

Figure 2 Apically attached neutrophil (PMN) exhibit intercellular adhesion molecule-1 (ICAM-1)- and Mac-1-dependent locomotion. (a, b) Following transepithelial migration (TEM), PMN were allowed to apically adhere to intestinal epithelial cells (IECs) grown in the bottom chamber of transwells. The number of PMN exhibiting locomotion (a) and the traveled distances (b) were visualized and quantified in the presence or absence of the appropriate IgG control, or anti-ICAM-1 and Mac-1 function-blocking antibodies (Abs; $20 \mu \mathrm{g} \mathrm{ml}^{-1}$ ), using phase-contrast time-lapse microscopy (Zeiss, Axiovert microscope) and ImageJ software. (c) PMN before (solid black line) and after TEM (dotted line) were analyzed for Mac-1 expression using flow cytometry. Representative flow diagram $(n=4)$ shows robust upregulation of Mac-1 on PMN surface after TEM. The filled area represents control IgG staining. (d) Image sequence depicting representative PMN exhibiting locomotion on T84 IECs in the absence (upper panels) and in the presence of Mac-1-blocking Ab (bottom panels). *Initial PMN position, white arrows track PMN movement. The dashed lines highlight the path traveled by PMN over $40 \mathrm{~min}$. Bar $=20 \mu \mathrm{m}$. (e) Movement trajectories (in $\mu \mathrm{m}$ ) of six representative PMN from three independent experiments over 40 min time periods on the apical surface of interferon- $\gamma$ (IFN $\gamma$ )-stimulated T84 IECs in the absence (upper panels) and in the presence of Mac-1-blocking Ab (bottom panels). $n=4$ independent experiments in duplicates, ${ }^{\star \star} P<0.01$, analysis of variance with Newman-Keuls multiple comparison test (a,b).

\section{Ligation of epithelial ICAM-1 triggers an MLCK-dependent increase in intestinal epithelial permeability}

We observed that apical upregulation of ICAM-1 significantly potentiated the effects of Mac-1-dependent PMN adhesion on the epithelial barrier (Figure 3a). We thus hypothesized that the PMN-induced increase in permeability of IFN $\gamma$-treated epithelial monolayers was due to PMN contact-mediated ICAM-1 clustering and an induction of ICAM-1-dependent signaling events, as previously described in vascular endothelium. ${ }^{30,31}$ T84 IEC monolayers on permeable supports were treated with IFN $\gamma$ to induce ICAM-1 expression, followed by the addition of crosslinking Abs to ICAM-1. We confirmed that Ab-mediated crosslinking induced ICAM-1 clustering (Supplementary Figure S2C). As shown in Figure 4a, Ab-mediated crosslinking of ICAM-1 resulted in a timedependent decrease in TER, which correlated with increased paracellular flux of fluorescein isothiocyanate (FITC)-dextran $(3 \mathrm{kDa}$, Figure $4 \mathbf{b})$. This effect was specific for ICAM-1, as crosslinking of other epithelial surface molecules, major histocompatibility complex-1 (MHC-1; Figure 4), and the known PMN ligand, CD55 (Supplementary Figure S5), had no significant effect on permeability. Consistent with the absence of ICAM-1 expression on unstimulated T84 IECs, application of crosslinking Abs to the apical surface of these IECs in the absence of IFN $\gamma$ stimulation had no effect on TER (Supplementary Figure S4A). Similarly, consistent with IFN $\gamma$-induced ICAM-1 localization on the apical epithelial membrane, application of crosslinking Abs to the basolateral aspect of epithelial monolayers had no significant effect on barrier function (Supplementary Figure S4B).

We have previously shown that early events in PMN TEM (at the level of the basolateral epithelial membrane) trigger MLCKmediated decreases in TER. ${ }^{9}$ MLCK has been shown to have a key role in regulating epithelial permeability by regulating contraction of the perijunctional actomyosin ring through myosin II regulatory light-chain phosphorylation. ${ }^{32}$ We thus asked whether enhanced IEC permeability after ligation of apically expressed ICAM-1 was MLCK dependent. Indeed, Ab-mediated crosslinking of ICAM-1 resulted in MLCK phosphorylation (Tyr 464), consistent with MLCK activation (Figure 4c). Such activation was accompanied by decreased apical brush boarder and the perijunctional F-actin (Figure 4d), indicative of actin cytoskeletal reorganization. Importantly, inhibition of ICAM-1 ligation-induced MLCK phosphorylation in T84 cells with ML-7 $\left(20 \mu \mathrm{m},{ }^{33}\right.$ Supplementary Figure S4C) prevented ICAM-1-induced F-actin reorganization (Figure 4d), the decrease in TER (Figure 4a), and the increase in paracellular dextran flux (Figure 4b). ML-7 treatment $(20 \mu \mathrm{M})$ alone had no effect on TER. Together, these data suggest that under inflammatory conditions, PMN contact with the apical surface of crypt epithelial cells triggers ICAM-1-dependent signaling events, resulting in enhanced permeability.

\section{Engagement of ICAM-1 on the apical epithelial membrane facilitates enhanced PMN TEM}

As ligation of apically expressed ICAM-1 increased epithelial permeability, we performed experiments to examine whether 

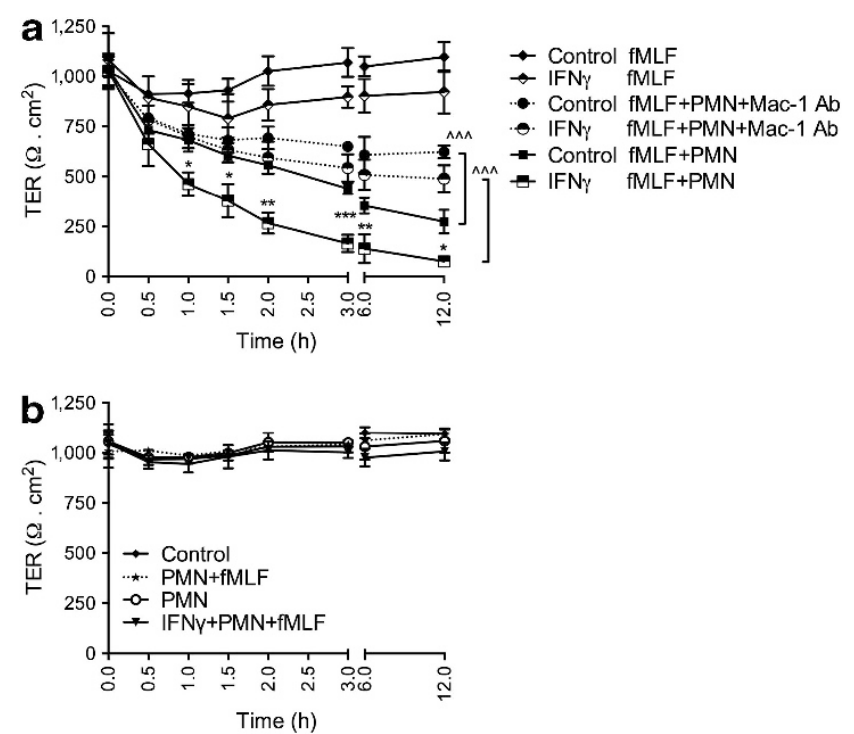

Figure 3 Neutrophil (PMN) interactions with the apical epithelial membrane compromise barrier function. (a) PMN $\left(2.5 \times 10^{5}\right.$ cells) were introduced to the apical surface of untreated (Control) or interferon- $\gamma$ $(\mathrm{IFN} \gamma)\left(100 \mathrm{U} \mathrm{ml}{ }^{-1}, 24 \mathrm{~h}\right)$-treated T84 intestinal epithelial cells (IECs) grown on permeable supports $(0.4 \mu \mathrm{m}$ filter size, prevent PMN transepithelial migration (TEM)), in the presence or absence of $\mathrm{N}$-formylMet-Leu-Phe (fMLF) (100 nM) and in the presence or absence of anti-Mac1 inhibitory antibody ( $\left.\mathrm{Ab} ; 20 \mu \mathrm{g} \mathrm{ml}^{-1}\right)$. The resulting changes in TER as an index of epithelial barrier function were measured at the indicated time points. PMN contact with apical epithelial surface resulted in a timedependent decrease in TER, which was partially reversed with inhibition of PMN-epithelial cell interactions. $N=4$ independent experiments in triplicates, ${ }^{*} P<0.05$, ${ }^{* *} P<0.01$, and ${ }^{* * *} P<0.001$ significantly different from Control + fMLF $+P M N, M \wedge P<0.001$ significantly different, analysis of variance with Newman-Keuls multiple comparison test. (b) PMNs were added in the vicinity of the apical membrane of unstimulated (control) or IFN $\gamma$-treated IECs (IFN $\gamma+$ PMN + fMLF) without direct contact (lower chamber of transwell setup) in the presence or absence of $\mathrm{MMLF}$ stimulation (100 nM). No changes in TER were observed, suggesting contact-dependent effects. $N=4$ independent experiments in triplicates.

ICAM-1-dependent alterations in epithelial barrier function would result in enhanced PMN TEM. We first investigated whether PMN engagement of ICAM-1 on the apical IEC membrane affected PMN TEM in the physiologically relevant basolateral-to-apical direction. ${ }^{34}$ In these experiments, PMN were stimulated to migrate across epithelial monolayers and the transmigrated cells were collected and re-applied for $1 \mathrm{~h}$ to the apical surface of new epithelial monolayers $\left(2.5 \times 10^{5} \mathrm{PMN}\right.$ per monolayer) with or without IFN $\gamma$ pretreatment. After $1 \mathrm{~h}$ of PMN-epithelial contact, the monolayers were washed free of adherent PMN and used for subsequent PMN TEM assays in the basolateral-to-apical direction. Apical introduction of PMN to IFN $\gamma$-treated, but not to untreated epithelial monolayers triggered a significant increase in PMN TEM (1.7-fold, Figure 5a). IFN $\gamma$ treatment alone or PMN presence near the apical surface, but without direct contact with monolayers, had no effect on PMN TEM (Figure 5a). In parallel experiments, PMN TEM was examined following specific Ab-mediated crosslinking of ICAM-1. Consistent with the effect of ICAM-1 crosslinking on IEC barrier function, Ab- mediated crosslinking of ICAM- 1 on IFN $\gamma$-treated T84 IECs significantly increased PMN TEM ( $1.9 \pm$ fold, Figure $5 \mathbf{b})$ compared with crosslinking of an apically expressed control molecule, MHC-1. As expected, application of ICAM-1 crosslinking protocols to untreated IEC monolayers had no significant effect on PMN TEM. Together, these findings suggest that PMN adherent to the apical (luminal) IEC membrane through engagement of ICAM-1 may trigger alterations in the epithelial barrier function and contribute to the regulation of PMN recruitment.

ICAM-1 crosslinking resulted in MLCK activation, suggesting actin-myosin contraction (Figure 4). Thus, we next examined the effects of MLCK inhibition and inhibition of F-actin contractile forces on PMN TEM following ICAM-1 crosslinking. Increased PMN TEM induced by ICAM-1 crosslinking was reversed when IECs were pretreated with either MLCK inhibitor ML-7 $\left(20 \mu \mathrm{M}, 1 \mathrm{~h}^{33}\right)$ or the myosin motor II inhibitor, Blebbistatin $\left(10 \mu \mathrm{M}, 1 \mathrm{~h},{ }^{35}\right.$ Figure $\left.5 \mathrm{c}\right)$. These findings suggest a role for actomyosin contraction downstream of ICAM-1 in regulating PMN TEM. Treatment with ML-7 or Blebbistatin alone had no effect on PMN TEM (Figure 5c).

\section{Ab-mediated ligation of ICAM-1 in murine intestinal lumen leads to MLCK-dependent increase in epithelial permeability and enhanced PMN recruitment}

We next performed in-vivo experiments to examine the effect of ICAM-1 ligation on intestinal epithelial barrier function and PMN recruitment, using a mouse intestinal loop model (Figure 6b). In this model, permeability of intact, bloodperfused segments of the small intestine was assessed after introduction of FITC-dextran to the intestinal lumen. As shown in Figure 6a, although ICAM-1 was not detected in unstimulated epithelium (upper panel), intraperitoneal (i.p.) administration of IFN $\gamma$ and TNF $\alpha$ (500 ng, $24 \mathrm{~h}$ ) resulted in a robust induction of ICAM-1 expression. In particular, induced ICAM-1 localized to apical regions of murine crypt IECs above Claudin-2 (Figure 6a, bottom panel). In parallel, we observed that cytokine treatment resulted in $\sim 2$-fold increase in permeability of the 3-kDa FITC-dextran (Figure 6c).

Importantly, introduction of ICAM crosslinking Abs, but not Abs to the control protein, MHC-1 into the lumen of cytokine-stimulated intestinal loops induced a further $\sim 1.5$-fold increase in permeability to dextran when compared with cytokine treatment alone (Figure 6c). To confirm that increases in intestinal epithelial permeability were mediated specifically by epithelial ICAM-1-induced signaling events, we used peptides derived from the cytoplasmic domain of ICAM-1 (ICAM-1 peptide) to inhibit the ability of ICAM-1 to mediate downstream signaling events. It has been previously shown in vascular endothelium that ICAM-1, but not control peptide, inhibited interactions between ICAM-1 and target proteins, thereby preventing ICAM-1-dependent signaling without affecting binding to extracellular leukocyte ligands. ${ }^{22,24}$ Addition of ICAM-1, but not the control peptide $\left(100 \mu \mathrm{g} \mathrm{ml}^{-1}\right.$, $30 \mathrm{~min}$ ), inhibited ICAM-1 ligation-induced increases in intestinal permeability (Figure 6c). Abs introduced intralumi- 


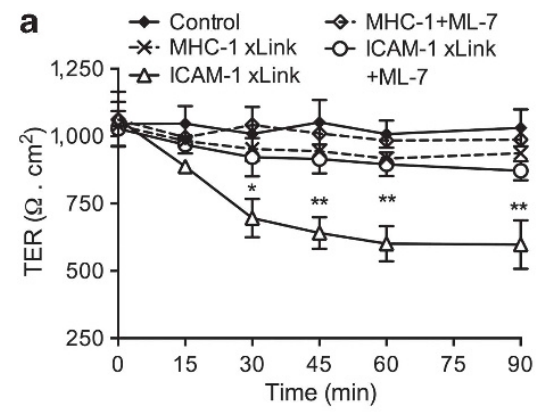

C
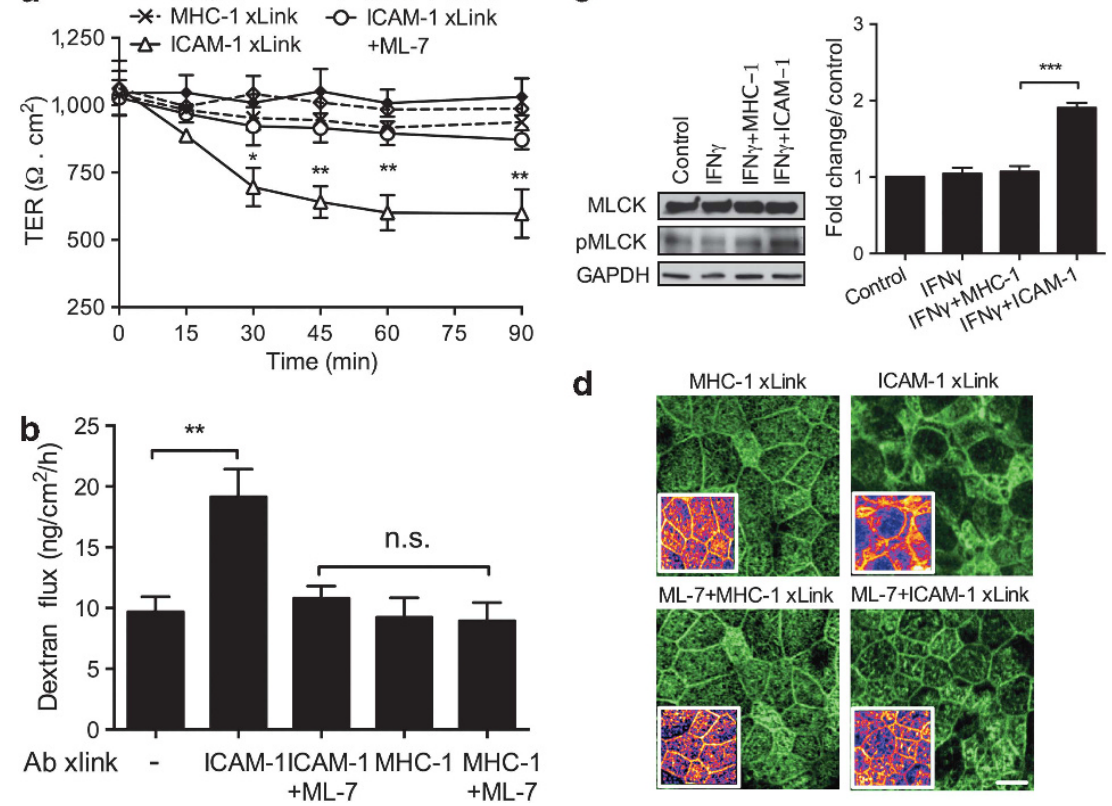

Figure 4 Antibody (Ab)-mediated crosslinking of intercellular adhesion molecule-1 (ICAM-1) leads to an myosin light-chain kinase (MLCK)-dependent increase in epithelial permeability. T84 intestinal epithelial cells (IECs) grown on permeable supports were stimulated with interferon- $\gamma$ (IFN $\gamma$ ) $\left(100 \mathrm{U} \mathrm{ml}^{-1}, 24 \mathrm{~h}\right)$ to induce ICAM-1 expression. ICAM-1 or control surface protein major histocompatibility complex-1 (MHC-1) were crosslinked by incubation with primary $\mathrm{Ab}\left(20 \mu \mathrm{g} \mathrm{ml}^{-1}, 60 \mathrm{~min}\right)$ followed by appropriate secondary antibodies $\left(20 \mu \mathrm{g} \mathrm{ml}^{-1}, 30 \mathrm{~min}\right)$. TER (a) and flux of fluorescein isothiocyanate (FITC)-dextran ( $3 \mathrm{kDa}$ ) (b) across T84 monolayers before and after ICAM-1 crosslinking were quantified at the indicated time points as an index of epithelial permeability. A pharmacological inhibitor of MLCK, ML-7 $(20 \mu \mathrm{M})$, was introduced an hour before initiation of the crosslinking protocols. ICAM-1 ligation resulted in decreased TER and increased flux of FITC-dextran, and was dependent on MLCK phosphorylation. (c) Representative western blottings and densitometric analysis (using ImageJ) show increased MLCK phosphorylation after ICAM-1 crosslinking, which was reversed with the addition of ML-7. (d) Confocal microscopy and immunofluorescence labeling were used to examine actin remodeling after crosslinking of apically expressed control receptor MHC-1, and specifically ICAM-1, in the presence or absence of ML-7 (20 $\mu \mathrm{M}$ ). Ab-mediated crosslinking of ICAM-1 but not MHC-1 resulted in decreased apical brush border and junctional actin. This effect was reversed in the presence of $\mathrm{ML}-7$. Bar $=50 \mu \mathrm{m} . N=4$ independent experiments in triplicates $(\mathbf{a}, \mathbf{b}),{ }^{\star} P<0.05,{ }^{\star \star} P<0.01$ significantly different from control $(\mathbf{a}),{ }^{\star \star} P<0.01$, ${ }^{\star \star \star} P<0.01$, n.s. not significant, (b,c), analysis of variance with Newman-Keuls multiple comparison test.
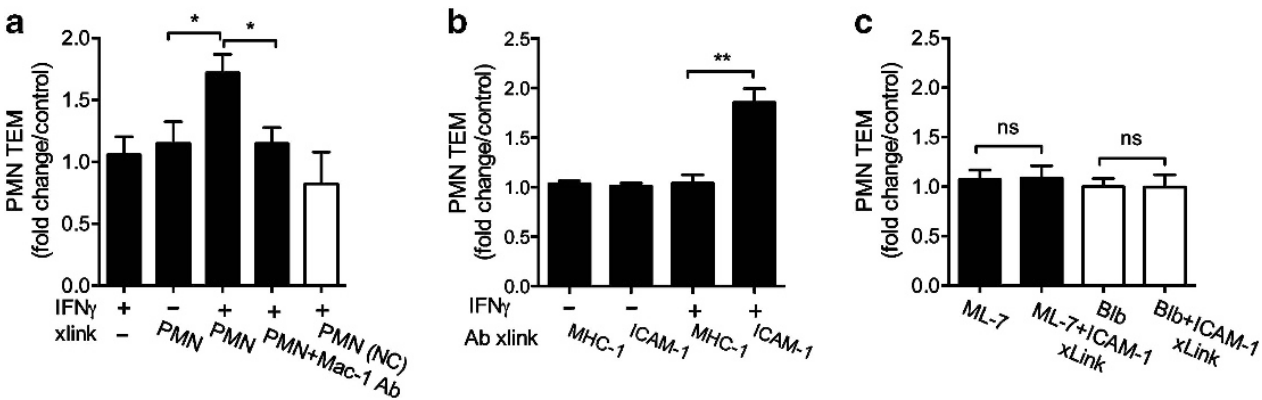

Figure 5 Engagement of apically expressed intercellular adhesion molecule-1 (ICAM-1) induces myosin light-chain kinase (MLCK)-dependent increase in neutrophil (PMN) transepithelial migration (TEM). PMN TEM in the basolateral-to-apical direction across untreated (control) or interferon- $\gamma$ (IFN $\gamma$ )-treated (to induce ICAM-1 expression) T84 monolayers was induced by a gradient of $N$-formyl-Met-Leu-Phe (fMLF) (100 nM).

(a) Transmigrated PMN were collected and introduced to the apical side of new T84 monolayers in the presence of fMLF (100 nM), or added to bottom chambers of transwells, facing the apical membrane, but without direct contact with the monolayers $\left(2.5 \times 10^{5} \mathrm{PMN}\right.$ per well, $\left.1 \mathrm{~h}\right)$. After washing off the apically adhered PMN, subsequent PMN TEM was quantified. PMN apical interactions specifically with IFN $\gamma$-treated T84 intestinal epithelial cells (IECs) significantly increased PMN TEM. This effect was reversed in the presence of anti-Mac-1 inhibitory antibody (Ab; $20 \mu \mathrm{gml}^{-1}$ ). For all conditions, the number of PMN that remained adherent to the apical epithelial membrane after washes was determined and subtracted from the total number of transmigrated PMN. (b) PMN TEM after Ab-mediated crosslinking of ICAM-1 or control protein (major histocompatibility complex-1 (MHC-1)) was quantified. Crosslinking of ICAM-1 but not MHC-1 on IFN $\gamma$-treated T84 cells significantly increased PMN TEM. (c) Control and IFN $\gamma$-treated T84 monolayers were preincubated with ML-7 (MLCK inhibitor, $20 \mu \mathrm{m}$ ) or Blebbistatin (myosin motor II inhibitor, $10 \mu \mathrm{m}$ ) alone or followed by ICAM-1 crosslinking. The effects of these inhibitors on ICAM-1 crosslinking induced increases in PMN TEM were quantified. Both inhibitors prevented ICAM-1 crosslinking-induced increases in PMN TEM. For all panels, the data presented as fold increase above PMN TEM across unstimulated T84 IECs. $N=4$ independent experiments in triplicates, ${ }^{\star} P<0.05$, ${ }^{\star \star} P<0.01$, n.s. not significant, analysis of variance with Newman-Keuls multiple comparison test. 
a

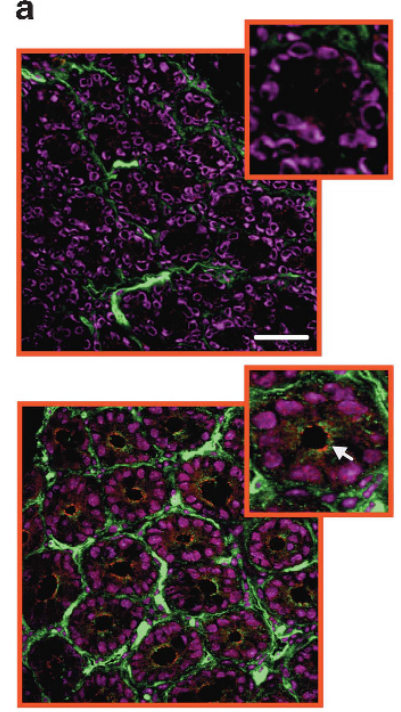

b

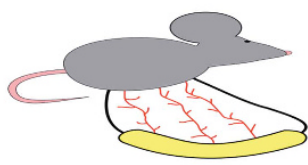

c

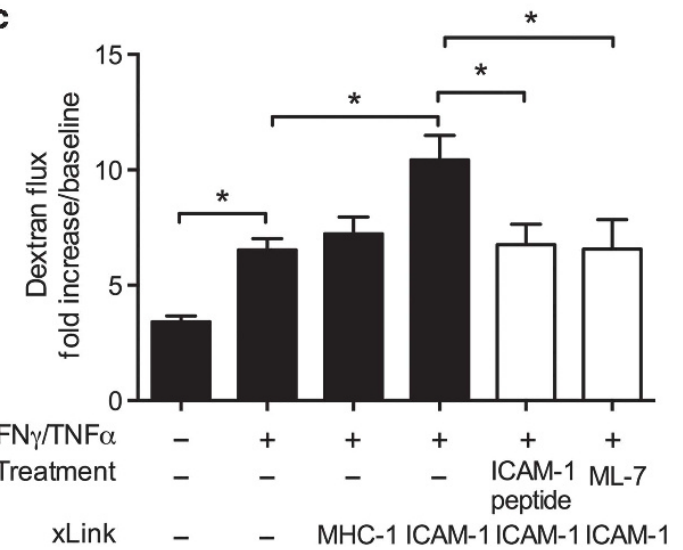

Figure 6 Engagement of intercellular adhesion molecule-1 (ICAM-1) in murine intestine in vivo leads to myosin light-chain kinase (MLCK)-dependent increase in intestinal permeability. To induce ICAM-1 expression, mice were injected with a mixture of interferon- $\gamma$ (IFN $\gamma$ ) and tumor necrosis factor- $\alpha$ (TNF $\alpha ; 500 \mathrm{ng}$ each, $24 \mathrm{~h}$, intraperitoneally (i.p.)). (a) OCT-frozen segments of mouse intestine were sectioned (7 $\mu \mathrm{m}$ wide) and immunofluorescently stained for ICAM-1 (green) and the tight junction protein Claudin-2 (red). Representative confocal images show apical induction of ICAM-1 expression (white arrow) after IFN $\gamma /$ TNF $\alpha$ treatment (bottom panel) compared with non-activated tissue (upper panel). Bar $=50 \mu \mathrm{m}$. (b) Cartoon depicts the mouse intestinal loop model as described in Methods. (c) In-vivo intestinal epithelial permeability was measured under the specified conditions as described in Methods. ICAM-1 tail peptide $\left(100 \mu \mathrm{m} \mathrm{ml}^{-1}\right)$ was introduced luminally 30 min before ICAM-1 crosslinking protocols. MLCK inhibitor ML-7 (20 $\mu \mathrm{M})$ was introduced by i.p. injection $2.5 \mathrm{~h}$ before ICAM-1 crosslinking. Increased fluorescein isothiocyanate (FITC)-dextran flux after antibody $(\mathrm{Ab})$ crosslinking of ICAM-1 was prevented with both the addition of ICAM-1 tail peptide and pretreatment with $\mathrm{ML}-7$. $N=4$ mice per condition, ${ }^{\star} P<0.05$, analysis of variance with Newman-Keuls multiple comparison test.

nally into intestinal loops (unstimulated and following IFN $\gamma / \mathrm{TNF} \alpha$ activation) were confirmed to not cross the epithelium, thus ruling out the potential indirect contribution of lamina propria cells to the observed effects (Supplementary Figure S6).

In further support of the role of MLCK in ICAM-1-mediated signaling, inhibition of MLCK activation using ML-7 $\left(1 \mathrm{mg} \mathrm{kg}^{-1}\right.$, i.p. $\left.{ }^{36}\right)$ before ICAM-1 crosslinking prevented the increase in permeability (Figure $\mathbf{6 c}$ ). These data demonstrate that increased intestinal epithelial permeability in vivo after ICAM-1 ligation is MLCK dependent.

As increased epithelial permeability is associated with enhanced PMN migration, we asked whether ligation of ICAM-1 would lead to enhanced PMN recruitment in vivo. Using the murine intestinal loop model, PMN infiltration into the intestinal mucosa and migration into the lumen was induced by luminal administration of chemoattractant CXCL1 $(1 \mu \mathrm{M}$ in $200 \mu \mathrm{l}$ Hank's balanced salt solution (HBSS) +$)$ and quantified by immunofluorescence labeling/confocal microscopy and analysis of lavaged fluid prepared on cytospins, and stained with Diff-Quik, respectively. In the absence of chemoattractant, PMNs were not detected in the intestinal epithelium or in the intestinal lumen (not shown); however, luminal administration of CXCL1 triggered significant PMN migration into the epithelial layer (Figure 7a) and accumulation in the intestinal lumen (Figure 7c). Consistent with the cytokine-induced increase in permeability, IFN $\gamma / \mathrm{TNF} \alpha$ treatment increased by $\sim 2.2$-fold the number of PMN in the intestinal epithelium, and $\sim 1.7$-fold in the lumen. Importantly, addition of ICAM-1 crosslinking Abs into the lumen of cytokine-treated intestinal loops further increased (over cytokine treatment alone) the number of PMN both in the epithelium $(\sim 1.6$-fold, Figure $7 \mathbf{a}, \mathbf{b})$ and in the lumen $(\sim 1.4-$ fold, Figure $7 \mathbf{c}, \mathbf{d}$ ). PMN (green) infiltrating the intestinal epithelium (red) are shown in representative images of tissue sections from cytokine-activated intestinal loops after ICAM-1 ligation (Figure 7b). Similarly, PMN that migrated into the lumen after ICAM-1 ligation are shown in representative images of lavage fluids from intestinal loops (Figure 7d).

Consistent with ICAM-1 ligation-induced increases in epithelial permeability, enhanced PMN migration into the intestinal lumen was dependent on ICAM-1-mediated signaling events and MLCK activation. Both intraluminal addition of ICAM-1 peptide, but not the control peptide (not shown; $100 \mu \mathrm{g} \mathrm{ml}^{-1}, 30 \mathrm{~min}$ ), and inhibition of MLCK (ML-7, $1 \mathrm{mg} \mathrm{kg}^{-1}$, i.p.) before Ab-mediated crosslinking of ICAM-1 completely inhibited ICAM-1 ligation-induced increases in PMN migration (Figure 7a,c). These findings suggest that engagement of ICAM-1 on the apical intestinal epithelial membrane under conditions of inflammation compromises the barrier function, thus facilitating enhanced PMN recruitment in vivo.

\section{DISCUSSION}

ICAM-1 expressed on the luminal aspect of vascular endothelium under inflammatory conditions ${ }^{37}$ mediates leukocyte 
a

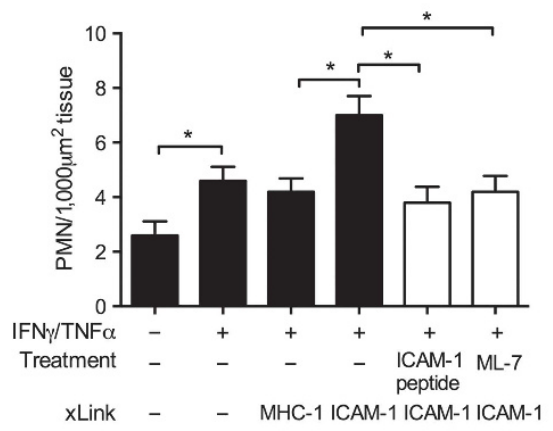

C
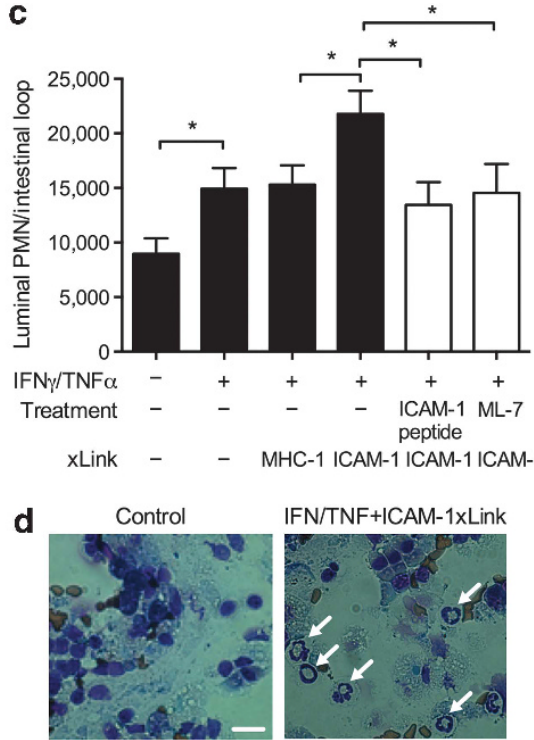

IFN/TNF+ICAM-1xLink

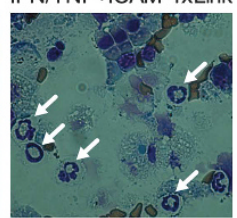

Figure 7 Engagement of intercellular adhesion molecule-1 (ICAM-1) in murine intestine in vivo leads to myosin light-chain kinase (MLCK)-dependent increase in neutrophil (PMN) recruitment. PMN transepithelial migration (TEM) in the murine intestinal loop model was induced by luminal introduction of CXCL1 (1 $\mu \mathrm{m}$ in $200 \mu \mathrm{l}$ Hank's balanced salt solution (HBSS) +). (a) PMN in the mucosal epithelia were quantified from cryosections of the intestinal loop immunofluorescently labeled for PMN (green) and F-actin (red). (b) Representative images show robust PMN infiltration after ICAM-1 crosslinking (right panel) compared with intestinal tissue without introduction of CXCL1, where PMN are localized inside the blood vessels (white arrow). Bar $=50 \mu \mathrm{m}$. (c) PMN that migrated into the lumen were isolated by lavage and quantified from cytospins stained with Diff-Quik. Data presented as percent of all cells in the lavage fluid. (d) Representative images of the cytospins depict transmigrated PMN in the intestinal luminal lavage after ICAM-1 crosslinking (right panel, PMN are indicated by white arrows). PMN are not detected in the intestinal lumen without the introduction of the chemoattractant (left panel). Bar $=10 \mu \mathrm{m} . N=4$ mice per condition, ${ }^{*} P<0.05$, analysis of variance with Newman-Keuls multiple comparison test.

migration across the endothelium in a luminal (apical)-tobasolateral direction. Engagement of ICAM-1 during early stages of the extravasation cascade and ICAM-1 clustering around migrating leukocytes ${ }^{23}$ is essential for efficient leukocyte transendothelial migration. Furthermore, PMN-mediated ligation of endothelial ICAM-1 triggers signaling events, resulting in cytoskeletal and junctional reorganization, thus priming the endothelium for leukocyte passage. ${ }^{24}$ In mucosal organs, it is now appreciated that ICAM-1 expression is induced under inflammatory conditions in bronchial, ${ }^{38}$ alveolar, ${ }^{39}$ and IEC cells. ${ }^{17}$ However, the localization of epithelial ICAM-1 is not in a position to mediate early adhesive interactions with migrating PMN. In fact, epithelialexpressed ICAM-1 localizes to the apical or luminal membrane so that migrating PMN that are recruited into the intestine would only encounter epithelial ICAM-1 after crossing the epithelium. Given these observations, the functional role for ICAM-1 in epithelial cells has not been clearly defined.

In the current work, we used complementary in-vitro and invivo approaches to show that engagement of ICAM-1 on the apical epithelial membrane by PMN or by Ab-mediated crosslinking resulted in MLCK-mediated actin reorganization, increased epithelial permeability, and enhanced PMN TEM. Thus far, regulation of PMN migration across epithelial layers has been largely attributed to PMN interactions with the basolateral aspects of epithelia ${ }^{9}$ and to $\mathrm{PMN}$-secreted proteaseinduced reorganization of epithelial junctional complexes during TEM. ${ }^{40}$ Our findings introduce a new mechanism, suggesting that under inflammatory conditions the presence of post-migrated PMN on the luminal epithelial surface and interactions with apically expressed epithelial ligands have regulatory effects on epithelial permeability and recruitment of PMN. Furthermore, under pathologic conditions characterized by persistence of large numbers of infiltrated PMN, such as that observed in active ulcerative colitis, sustained engagement of ICAM-1 could enhance PMN TEM, thus contributing to PMNassociated epithelial injury. However, such mechanisms may also be beneficial in providing a robust pro-inflammatory response that aids in clearance of invading microorganisms by promoting PMN recruitment. These findings are summarized in a model depicted in Figure 8.

Exposure of T84 IECs to IFN $\gamma$ induced ICAM-1 expression, resulting in enhanced PMN retention on the apical epithelial membrane after TEM. Intriguingly, apically associated PMN after completing TEM were observed to exhibit ICAM-1- and Mac-1-dependent apical locomotion, as has been previously described for vascular endothelium. ${ }^{24}$ In blood vessels, luminal locomotion of PMN is an essential mechanism used by adherent PMN to locate the specific endothelial regions that can support PMN transmigration. In contrast, in the intestine or the lung, PMN luminal locomotion would occur post-TEM, thus clearly serving a different purpose. Although it has been suggested that PMN can migrate through mucus, ${ }^{41}$ we speculate that PMN migrating along the luminal IEC surface would be more efficient in reaching the specific sites of inflammation. Moreover, PMN exhibiting locomotion may 


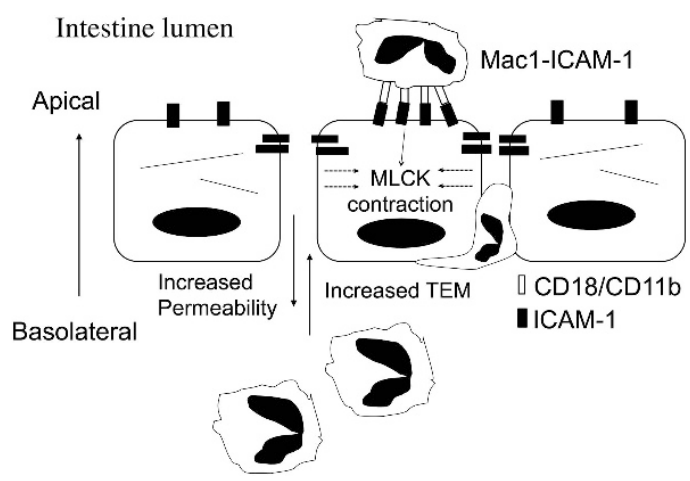

Figure 8 Hypothetical model depicting neutrophil (PMN) engagement of intercellular adhesion molecule-1 (ICAM-1) on the apical epithelial surface and alteration of epithelial barrier function, thereby facilitating enhanced PMN transepithelial migration (TEM). PMN adhered to apically expressed ICAM-1 after completing TEM exhibit luminal locomotion and ligation of ICAM-1. Engagement of ICAM-1 triggers MLCK activation, reorganization of the actin cytoskeleton, and cell contraction, leading to increased paracellular permeability. As a result of the compromised barrier function, the number of PMN migrating across epithelial monolayers is significantly enhanced.

lead to clustering of apically expressed ligands, which in the case of ICAM-1 results in initiation of signaling events that alter epithelial barrier function, thereby facilitating enhanced PMN recruitment during acute inflammatory responses.

In support of this, we found that PMN interactions with the luminal epithelial surface following stimulation with IFN $\gamma$ resulted in increased epithelial permeability. These changes were PMN-IEC contact dependent, as they were reversed on inhibition of PMN adhesion/locomotion by anti-Mac-1 function-blocking Abs.

Several other apical epithelial ligands have been identified as being important for PMN-IEC interactions. For example, CD55 has been reported to promote PMN detachment, ${ }^{18}$ thus potentially competing with ICAM-1 for PMN interactions. $\mathrm{CD} 44$ is another apical epithelial ligand that is markedly upregulated during inflammation. ${ }^{19,42}$ Although it has been implicated in promoting PMN detachment ${ }^{19}$ similar to ICAM1, CD44 has also been shown to act as a signaling receptor through interactions with the actin cytoskeleton, thus contributing to tumor metastasis. ${ }^{43}$ Although we established that ligation of CD55 had no effect on epithelial permeability, whether this is true for other apically expressed PMN ligands should be explored in future studies.

We also demonstrate the importance of actomyosin dynamics in the regulation of PMN TEM. Specifically, ICAM-1 ligation induced MLCK activation and MLCK-dependent reorganization of the actin cytoskeleton, resulting in increased epithelial permeability and PMN TEM. These effects were likely due to increased actomyosin contractility, as MLCK has been previously shown to mediate cell contraction through actin cytoskeleton remodeling and activation of actomyosin contraction. ${ }^{33,44}$ Inhibition of both MLCK activation and myosin motor II mediated actomyosin contraction, prevented ICAM-1 ligation-induced increases in permeability, and PMN TEM
(Figures $\mathbf{4 a , b}$ and $\mathbf{5 c}$ ). It was previously shown that ICAM-1 associates with the actin cytoskeleton through interactions of its cytosolic domain (ICAM-1 tail) with adapter proteins. ${ }^{45}$ Inhibition of these interactions in vivo, using cell-permeable peptides constituting the cytoplasmic domain of ICAM-1 also prevented ICAM-1 ligation-induced increases in permeability and in PMN TEM, confirming the specificity of the observed effects to ICAM-1-mediated signaling. Interestingly, we have also shown that stimulus-dependent PMN contact with the basolateral epithelial surface, which was induced by a chemoattractant gradient, resulted in MLCK-dependent alterations in the epithelial barrier function. ${ }^{9}$ These effects were induced downstream of basolaterally expressed proteaseactivated receptors- 1 and -2 . Together with current observations, these findings suggest that similar cellular events, such as MLCK activation that are capable of modulating epithelial function, can be induced through engagement of ligands localized to either basolateral or apical epithelial surfaces during both early and late stages of PMN TEM.

In summary, these findings highlight an important role for post-migrated PMN on the luminal epithelial surface and their interactions with apical epithelial ligands in the regulation of epithelial barrier function during inflammation. Impaired epithelial barrier and increased numbers of infiltrating PMN are ultimately linked to mucosal injury as often observed in inflammatory disorders of the mucosal surfaces.

\section{METHODS}

Cells. Human T84 IECs were grown in Dulbecco's modified Eagle's medium-F12 50:50 (Sigma, St Louis, MO) with supplements as previously described. ${ }^{34}$ For PMN isolation, human blood was drawn and handled according to protocols for the protection of human subjects, as approved by the Emory University Hospital Institutional Review Board. PMN were isolated by density gradient centrifuga$\operatorname{tion}^{34,46}$ and were used in experiments within $2 \mathrm{~h}$ of isolation.

Antibodies and reagents. The following Abs were used: functionblocking anti-human ICAM-1 (15.2) from Serotec (Raleigh, NC), antimouse ICAM-1mAb (YN1/1.7.4) and anti-Ly-6G conjugated to Alexa 488 from eBioscience (San Diego, CA), anti-CD11b/CD18 mAb (CBRM1/29) has been described, ${ }^{47}$ anti-Mouse-Alexa488 and antirabbit-Alexa555 from Invitrogen (Carlsbad, CA), anti-MHC Class-I (1.B.548) from Abcam (Cambridge, MA), isotype control IgG1 from BD Biosciences (San Jose, CA), rabbit polyclonal anti-MLCK from ProteinTech (Chicago IL), and anti-phospho MLCK from Santa Cruz (Santa Cruz, CA). Horseradish peroxidase-conjugated anti-mouse and anti-rabbit IgGs were obtained from Jackson Immunoresearch (West Grove, PA). ABTS (2,2'-azinobis- 3-ethylbenzothiazoline-6-sulfonic acid), HBSS with $\mathrm{Ca}^{2+}$ and $\mathrm{Mg}^{2+}$ (HBSS + ), and HBSS without $\mathrm{Ca}^{2+}$ and $\mathrm{Mg}^{2+}$ (HBSS - ), and Dulbecco's modified Eagle's medium including media supplements and chemotactic peptide fMLF, mouse IFN $\gamma$, and TNF $\alpha$ were obtained from Sigma. Fluorescein-labeled dextran $(3 \mathrm{kDa})$ were obtained from Molecular Probes (Eugene, OR).

Western blotting. Epithelial cells were prepared for western blot analysis using standard techniques, as previously described. ${ }^{19}$

Flow cytometry. Adherent epithelial cell monolayers were trypsinized (Trypsin-EDTA, Sigma), washed, and stained for ICAM-1 (15.2 FITC, $\left.5 \mu \mathrm{g} \mathrm{ml}^{-1}\right)$. PMN before and after TEM were stained for Mac-1 (ICRF44, $20 \mu \mathrm{l}$ per test). Cell samples were analysed using FACS Calibur (San Jose, CA) and FlowJo software (Ashland, OR). 
Cell adhesion assay. PMN were incubated with confluent monolayers in 24 -well tissue culture plates $\left(2.5 \times 10^{5}\right.$ cell per well in $\mathrm{H}+$, $1 \mathrm{~h}, 37^{\circ} \mathrm{C}$ ). Following three consecutive washes, the remaining adherent PMN were quantified in 10 randomly selected fields of view, in triplicate, for each experimental condition.

Immunofluorescence microscopy. IEC monolayers were fixed/ permeabilized (95\% ethanol) and incubated with appropriate primary Abs $\left(10 \mu \mathrm{g} \mathrm{ml}^{-1}\right.$, overnight at $\left.4{ }^{\circ} \mathrm{C}\right)$ followed by fluorescently labeled secondary Abs $\left(1 \mu \mathrm{g} \mathrm{ml}^{-1}, 1 \mathrm{~h}\right.$ at room temperature). Nuclei were stained with ToPro-3 iodide (Invitrogen). For mouse tissue, segments of mouse intestine were snap frozen in OCT, $7-\mu \mathrm{m}$-thick sections were cut on a freezing microtome, and mounted on slides for immunofluorescence labeling. All images were acquired on a LSM510 confocal microscope (Carl Zeiss, Thornwood, NY) with Plan-Neofluor $\times 60$ and $\times 40$ objectives.

Mouse intestinal loop model. Male C57BL6J mice (Jackson Laboratories, age 11-15 weeks) were maintained under specific pathogen-free conditions at the Emory Division of Animal Resources facilities. When indicated, inflammation was induced by i.p. injection of mouse recombinant TNF $\alpha$ and IFN $\gamma(0.5 \mu$ g each $)$ in $0.25 \mathrm{ml}$ saline $24 \mathrm{~h}$ before the start of the surgical preparation. Animals were anesthetized by subcutaneous intramuscular injection of ketamine and xylazine mixture at doses of 100 and $5 \mathrm{mg} \mathrm{kg}^{-1}$, respectively. A midline abdominal incision was made and a $4-\mathrm{cm}$ loop of small intestine was exteriorized and clipped at proximal and distal ends (Figure 6b). After luminal administration of the desired treatment, the excised loops were reinserted into the peritoneal cavity for the duration of the experimental procedures. At the end of all experimental procedures, animals were killed via rapid cervical dislocation. All animal protocols were reviewed and approved by the Institutional Animal Care and Use Committee of Emory University.

PMN TEM assays. For in-vivo PMN migration experiments, exteriorized intestinal loops were injected with $1 \mu \mathrm{M}$ PMN chemoattractant CXCL1 (KC) in $200 \mu \mathrm{l} \mathrm{HBSS}+$. Ninety minutes later, intestinal loops were isolated and lavaged twice with $200 \mu \mathrm{l} \mathrm{HBSS}+$. PMN in the lavaged fluid (PMN that migrated into the intestinal lumen) were quantified on $100 \mu \mathrm{l}$ cytospins stained with Diff-Quik (Dade Behring, Newark, DE). In-vitro PMN TEM assays were performed in the physiologically relevant basolateral-to-apical direction and quantified by assaying for the PMN azurophilic granule protein myeloperoxidase, as previously described. ${ }^{34}$ Apically associated PMN were collected by centrifugation (500 r.p.m., $3 \mathrm{~min}$ ) and assayed for myeloperoxidase. When indicated, IECs were pretreated with IFN $\gamma\left(100 \mathrm{U} \mathrm{ml}^{-1}\right)$ for $24 \mathrm{~h}$ to induce ICAM-1 expression.

PMN locomotion on epithelial cells. Following migration across T84 IEC monolayers, PMN adhered to the apical membrane of confluent T84 IEC monolayers grown in the bottom chamber of the transwell setup were visualized using phase-contrast time-lapse microscopy (Zeiss Axiovert microscope). PMN locomotion (\% locomoting cells, distances, and velocity) was quantified using ImageJ software (National Institute of Technology, Bethesda, MD).

Epithelial permeability assays. For in-vivo dextran flux assay, exteriorized intestinal loops were injected with FITC-dextran $(3 \mathrm{kDa}$, $1 \mathrm{mg} \mathrm{ml}^{-1}$ in $200 \mu \mathrm{l}$ saline) and reinserted into the peritoneal cavity. sixty minutes later, fluorescence intensity in whole blood (obtained through cardiac puncture) was analyzed on a fluorescence plate reader (Fluostar Galaxy, BMG LabTech, Ortenberg, Germany), using excitation/emission wavelengths of $480 / 520 \mathrm{~nm}$ as previously described. ${ }^{48}$ For in-vitro dextran flux assay, $10 \mu \mathrm{g} \mathrm{ml}^{-1}$ fluorescein-labeled dextran $(3 \mathrm{kDa})$ was added apically to IEC monolayers grown on permeable supports. Samples were taken from the bottom chambers at the indicated time points and fluorescence intensity in the samples was measured.
ICAM-1 crosslinking. In-vitro, primary anti-ICAM-1 (clone 15.2, $\left.20 \mu \mathrm{g} \mathrm{ml}^{-1}, 1 \mathrm{~h}\right)$ followed by secondary crosslinking Abs $\left(20 \mu \mathrm{g} \mathrm{ml}^{-1}\right.$, $30 \mathrm{~min}$ ) were added apically to control/IFN $\gamma$ pre-exposed epithelial monolayers. Where specified, IECs were preincubated with ML-7 $(20 \mu \mathrm{M})$ and blebbistatin $(10 \mu \mathrm{M})$ for $1 \mathrm{~h}$ at $37^{\circ} \mathrm{C}$. In-vivo, before the introduction of FITC-dextran or CXCL1, isolated intestinal loops were cannulated at proximal and distal ends with $0.76-\mathrm{mm}$ internal diameter polyethylene tubing, filled with ICAM-1 (YN1/1.7.4) or MHC-1 (1.B.548) Ab solutions $\left(50 \mu \mathrm{g} \mathrm{ml}^{-1}\right.$ in $200 \mu \mathrm{l}$ saline warmed to $37^{\circ} \mathrm{C}$, $1 \mathrm{~h})$, flushed with saline, and refilled with secondary crosslinking Abs $\left(50 \mu \mathrm{g} \mathrm{ml}^{-1}\right.$ in $200 \mu \mathrm{l}$ saline) for an additional $30 \mathrm{~min}$. Where specified, ICAM-1 cytoplasmic domain peptide (13 C-terminal amino acids of mouse ICAM-1 (QRKIRIYKLQQAQ) attached to penetratin (RQIKIWFQNRRMKWKK), ${ }^{24} 100 \mu \mathrm{g} \mathrm{ml}^{-1}$ ) or control peptide (an irrelevant sequence from rat rhodopsin, CKPMSNFRFGENH) was introduced intraluminally $30 \mathrm{~min}$ before ICAM-1 crosslinking. The MLCK inhibitor ML-7 (1 mg kg-1) was introduced by i.p. injection $2.5 \mathrm{~h}$ before the initiation of surgical protocols.

Statistics. Statistical significance was assessed by Student's $t$-test or by one-way analysis of variance with Newman-Keuls multiple comparison test using Graphpad Prism (V4.0, La Jolla, CA). Statistical significance was set at $P<0.05$. For all experiments, the data are shown as \pm s.e.m.

SUPPLEMENTARY MATERIAL is linked to the online version of the paper at http://www.nature.com/mi

\section{ACKNOWLEDGMENTS}

We thank Emory DDRDC core facility for culturing IEC cell lines (supported $\mathrm{NIH}$ DK064399). We thank Dr Ingrid H Sarelius for kindly providing the ICAM-1 and control peptides. This work was supported in part by grants from the NIH (DK072564, DK061379, and DK079392 to CP, and DK055679 and DK059888 to AN) and CDA from the Crohn's and Colitis Foundation of America to RS.

\section{DISCLOSURE}

The authors declare no conflict of interest.

(c) 2014 Society for Mucosal Immunology

\section{REFERENCES}

1. Matute-Bello, G., Frevert, C.W. \& Martin, T.R. Animal models of acute lung injury. Am. J. Physiol. Lung Cell Mol. Physiol. 295, L379-L399 (2008).

2. Grommes, J. \& Soehnlein, O. Contribution of neutrophils to acute lung injury. Mol. Med. 17, 293-307 (2011).

3. De Lisle, R.C., Mueller, R. \& Boyd, M. Impaired mucosal barrier function in the small intestine of the cystic fibrosis mouse. J. Pediatr. Gastroenterol. Nutr. 53, 371-379 (2011).

4. Schmitz, H. et al. Altered tight junction structure contributes to the impaired epithelial barrier function in ulcerative colitis. Gastroenterology 116, 301-309 (1999).

5. Ma, T.Y. Intestinal epithelial barrier dysfunction in Crohn's disease. Proc. Soc. Exp. Biol. Med. 214, 318-327 (1997).

6. Ley, K., Laudanna, C., Cybulsky, M.I. \& Nourshargh, S. Getting to the site of inflammation: the leukocyte adhesion cascade updated. Nat. Rev. Immunol. 7, 678-689 (2007).

7. Zen, K., Liu, Y., Cairo, D. \& Parkos, C.A. CD11b/CD18-dependent interactions of neutrophils with intestinal epithelium are mediated by fucosylated proteoglycans. J. Immunol. 169, 5270-5278 (2002).

8. Parkos, C.A., Delp, C., Arnaout, M.A. \& Madara, J.L. Neutrophil migration across a cultured intestinal epithelium. Dependence on a CD11b/CD18mediated event and enhanced efficiency in physiological direction. J. Clin. Invest. 88, 1605-1612 (1991).

9. Chin, A.C., Lee, W.Y., Nusrat, A., Vergnolle, N. \& Parkos, C.A. Neutrophilmediated activation of epithelial protease-activated receptors-1 and -2 regulates barrier function and transepithelial migration. J. Immunol. 181, 5702-5710 (2008). 
10. Zemans, R.L., Colgan, S.P. \& Downey, G.P. Transepithelial migration of neutrophils: mechanisms and implications for acute lung injury. Am. J. Respir. Cell Mol. Biol. 40, 519-535 (2009).

11. Mumy, K.L. \& McCormick, B.A. The role of neutrophils in the event of intestinal inflammation. Curr. Opin. Pharmacol. 9, 697-701 (2009).

12. Fournier, B.M. \& Parkos, C.A. The role of neutrophils during intestinal inflammation. Mucosal. Immunol. 5, 354-366 (2012).

13. Liu, Y. et al. Signal regulatory protein (SIRPalpha), a cellular ligand for CD47, regulates neutrophil transmigration. J. Biol. Chem. 277, 10028-10036 (2002).

14. Xavier, R.J. \& Podolsky, D.K. Unravelling the pathogenesis of inflammatory bowel disease. Nature 448, 427-434 (2007).

15. Louis, N.A., Hamilton, K.E., Kong, T. \& Colgan, S.P. HIF-dependent induction of apical CD55 coordinates epithelial clearance of neutrophils. FASEB J. 19, 950-959 (2005).

16. Leir, S.H., Holgate, S.T. \& Lackie, P.M. Inflammatory cytokines can enhance CD44-mediated airway epithelial cell adhesion independently of CD44 expression. Am. J. Physiol. Lung Cell Mol. Physiol. 285, L1305-L1311 (2003).

17. Kaiserlian, D., Rigal, D., Abello, J. \& Revillard, J.P. Expression, function and regulation of the intercellular adhesion molecule-1 (ICAM-1) on human intestinal epithelial cell lines. Eur. J. Immunol. 21, 2415-2421 (1991).

18. Lawrence, D.W. et al. Antiadhesive role of apical decay-accelerating factor (CD55) in human neutrophil transmigration across mucosal epithelia. J. Exp. Med. 198, 999-1010 (2003).

19. Brazil, J.C. etal. Neutrophil migration across intestinal epithelium: evidence for a role of CD44 in regulating detachment of migrating cells from the luminal surface. J. Immunol. 185, 7026-7036 (2010).

20. Huang, G.T., Eckmann, L., Savidge, T.C. \& Kagnoff, M.F. Infection of human intestinal epithelial cells with invasive bacteria upregulates apical intercellular adhesion molecule-1 (ICAM)-1) expression and neutrophil adhesion. J. Clin. Invest. 98, 572-583 (1996).

21. Ponta, H., Sherman, L. \& Herrlich, P.A. CD44: from adhesion molecules to signalling regulators. Nat. Rev. Mol. Cell Biol. 4, 33-45 (2003).

22. Yang, L. et al. ICAM-1 regulates neutrophil adhesion and transcellular migration of TNF-alpha-activated vascular endothelium under flow. Blood 106, 584-592 (2005).

23. Carman, C.V. \& Springer, T.A. A transmigratory cup in leukocyte diapedesis both through individual vascular endothelial cells and between them. J. Cell Biol. 167, 377-388 (2004).

24. Sumagin, R. \& Sarelius, I.H. Intercellular adhesion molecule-1 enrichment near tricellular endothelial junctions is preferentially associated with leukocyte transmigration and signals for reorganization of these junctions to accommodate leukocyte passage. J. Immunol. 184, 5242-5252 (2010).

25. Lawson, C. \& Wolf, S. ICAM-1 signaling in endothelial cells. Pharmacol. Rep. 61, 22-32 (2009).

26. Vainer, B., Nielsen, O.H. \& Horn, T. Comparative studies of the colonic in situ expression of intercellular adhesion molecules (ICAM-1, -2, and -3), beta2 integrins (LFA-1, Mac-1, and p150,95), and PECAM-1 in ulcerative colitis and Crohn's disease. Am. J. Surg. Pathol. 24, 1115-1124 (2000).

27. Parkos, C.A. et al. Expression and polarization of intercellular adhesion molecule-1 on human intestinal epithelia: consequences for CD11b/CD18mediated interactions with neutrophils. Mol. Med. 2, 489-505 (1996).

28. Sumagin, R., Prizant, H., Lomakina, E., Waugh, R.E. \& Sarelius, I.H. LFA-1 and Mac-1 define characteristically different intralumenal crawling and emigration patterns for monocytes and neutrophils in situ. J. Immunol. 185, 7057-7066 (2010).

29. Ross, E.A. et al. Interaction between integrin alpha9beta1 and vascular cell adhesion molecule-1 (VCAM-1) inhibits neutrophil apoptosis. Blood 107, 1178-1183 (2006).
30. Sumagin, R., Lomakina, E. \& Sarelius, I.H. Leukocyte-endothelial cell interactions are linked to vascular permeability via ICAM-1mediated signaling. Am. J. Physiol. Heart Circ. Physiol. 295, H969-H977 (2008).

31. Sumagin, R., Kuebel, J.M. \& Sarelius, I.H. Leukocyte rolling and adhesion both contribute to regulation of microvascular permeability to albumin via ligation of ICAM-1. Am. J. Physiol. Cell Physiol. 301, C804-C813 (2011).

32. Cunningham, K.E. \& Turner, J.R. Myosin light chain kinase: pulling the strings of epithelial tight junction function. Ann. N. Y. Acad. Sci. 1258, 34-42 (2012).

33. Turner, J.R. et al. Physiological regulation of epithelial tight junctions is associated with myosin light-chain phosphorylation. Am. J. Physiol. 273, C1378-C1385 (1997).

34. Parkos, C.A., Colgan, S.P., Delp, C., Arnaout, M.A. \& Madara, J.L. Neutrophil migration across a cultured epithelial monolayer elicits a biphasic resistance response representing sequential effects on transcellular and paracellular pathways. J. Cell Biol. 117, 757-764 (1992).

35. Ivanov, A.I., Hunt, D., Utech, M., Nusrat, A. \& Parkos, C.A. Differential roles for actin polymerization and a myosin $\|$ motor in assembly of the epithelial apical junctional complex. Mol. Biol. Cell 16, 2636-2650 (2005).

36. Wu, C.C., Lu, Y.Z., Wu, L.L. \& Yu, L.C. Role of myosin light chain kinase in intestinal epithelial barrier defects in a rat model of bowel obstruction. BMC Gastroenterol. 10, 39 (2010).

37. Sumagin, R. \& Sarelius, I.H. TNF-alpha activation of arterioles and venules alters distribution and levels of ICAM-1 and affects leukocyte-endothelial cell interactions. Am. J. Physiol. Heart Circ. Physiol. 291, H2116-H2125 (2006).

38. Chan, S.C. et al. Upregulation of ICAM-1 expression in bronchial epithelial cells by airway secretions in bronchiectasis. Respir. Med. 102, 287-298 (2008).

39. Madjdpour, C. et al. Lipopolysaccharide induces functional ICAM-1 expression in rat alveolar epithelial cells in vitro. Am. J. Physiol. Lung Cell Mol. Physiol. 278, L572-L579 (2000).

40. Ginzberg, H.H. et al. Neutrophil-mediated epithelial injury during transmigration: role of elastase. Am. J. Physiol. Gastrointest. Liver Physiol. 281, G705-G717 (2001).

41. Parkhurst, M.R. \& Saltzman, W.M. Leukocytes migrate through threedimensional gels of midcycle cervical mucus. Cell. Immunol. 156, 77-94 (1994).

42. Mackay, C.R. et al. Expression and modulation of CD44 variant isoforms in humans. J. Cell Biol. 124, 71-82 (1994).

43. Mathew, J. et al. CD44 is expressed in hepatocellular carcinomas showing vascular invasion. J. Pathol. 179, 74-79 (1996).

44. Scott, K.G., Meddings, J.B., Kirk, D.R., Lees-Miller, S.P. \& Buret, A.G. Intestinal infection with Giardia spp. reduces epithelial barrier function in a myosin light chain kinase-dependent fashion. Gastroenterology 123, 1179-1190 (2002).

45. Yang, L. et al. Endothelial cell cortactin coordinates intercellular adhesion molecule-1 clustering and actin cytoskeleton remodeling during polymorphonuclear leukocyte adhesion and transmigration. J. Immunol. 177, 6440-6449 (2006).

46. Chin, A.C. et al. CD47 and TLR-2 cross-talk regulates neutrophil transmigration. J. Immunol. 183, 5957-5963 (2009).

47. Balsam, L.B., Liang, T.W. \& Parkos, C.A. Functional mapping of CD11b/ CD18 epitopes important in neutrophil-epithelial interactions: a central role of the I domain. J. Immunol. 160, 5058-5065 (1998).

48. Laukoetter, M.G. et al. JAM-A regulates permeability and inflammation in the intestine in vivo. J. Exp. Med. 204, 3067-3076 (2007). 